\title{
Psychological distress and quality of life of Malaysian infertile couples
}

\author{
Noorfaizah Ibrahim ${ }^{1}$, Salina Mohamed ${ }^{2}$, Zaliha Ismail ${ }^{3}$, Mohd Razali Salleh ${ }^{2 *}$
}

\begin{abstract}
${ }^{1}$ Department of Psychiatry, Hospital Sultan Ismail, Johor Bahru, Malaysia
${ }^{2}$ Department of Psychiatry, ${ }^{3}$ Department of Population Health and Preventive Medicine, Faculty of Medicine, University Technology MARA, Shah Alam, Malaysia
\end{abstract}

Received: 08 October 2020

Revised: 03 December 2020

Accepted: 04 December 2020

\section{*Correspondence:}

Dr. Mohd Razali Salleh,

E-mail: razali6403@uitm.edu.my

Copyright: ( ) the author(s), publisher and licensee Medip Academy. This is an open-access article distributed under the terms of the Creative Commons Attribution Non-Commercial License, which permits unrestricted non-commercial use, distribution, and reproduction in any medium, provided the original work is properly cited.

\begin{abstract}
Background: Infertility is an intense situation experienced by couples that causes emotional discomfort and worsening of their quality of life (QoL). The husband and wives of the infertile couples may reacted differently to stress.

Methods: This is a cross-sectional study involving 100 infertile couples attending fertility clinics. Cases were selected by systematic random sampling. The severity of depression, anxiety and stress was measured using the Depression, Anxiety and Stress Scale (DASS-21) and QoL was assessed with the Fertility Quality of Life (FertiQoL) questionnaire.

Results: Multivariate analysis revealed that depression, anxiety and stress-related difficulties were reported at a higher level by wives $(\mathrm{p}<0.001)$. Total Ferti QoL scores were significantly higher in husbands $(\mathrm{p}<0.001)$. The female gender (wives) was the only significant variable associated with the severity of all three DASS-21 sub-scale scores (p $<0.001)$ and considered as a high-risk factor for psychological distress. Poor Qol were significantly associated with wives $(p<0.001)$, male cause of infertility $(p=0.004)$, primary infertility $(p=0.022)$ and previous history of receiving intrauterine insemination (IUI) $(\mathrm{p}=0.020)$.

Conclusions: The wives of the infertile couples were at higher risk of developing psychological distress and had poor QoL than their husbands. The severity of the anxiety, depression and stress were adversely affected the QoL.
\end{abstract}

Keywords: Gender differences, Infertility, Quality of life, Psychological distress

\section{INTRODUCTION}

\section{Infertility in global perspective}

The term 'infertility' is used to describe a condition in which a woman is unable to attain a pregnancy after 12 months or more of regular unprotected sexual intercourse. ${ }^{1}$ According to the World Health Organization (WHO), quality of life (QoL) is described as how a person perceives his or her position in life based on their own value system and culture. ${ }^{2}$ Couples without reproductive problems who practice regular sex without birth control usually have an $85-90 \%$ likelihood of pregnancy within 1 year. ${ }^{3}$ Sexton et al estimated that $10-$ $15 \%$ of married couples of child-bearing age have severe difficulty in achieving pregnancy. ${ }^{4}$

An individual who marries at a later age, has higher educational achievement and occupational status is more at risk of developing infertility.

\section{Socio-cultural factors and gender differences}

Females are more likely to suffer from psychological disturbances, especially in developing countries, where the female is mostly believed to be the reason for the 
couple's inability to conceive. ${ }^{5}$ The psychological impact of infertility may range from an inferiority complex and stress, to interpersonal relationships, to major depression and anxiety. ${ }^{6}$ Emotional and psychological distress is the main contributor to a low QoL in infertile couples, as its associated with anxiety and depression. ${ }^{7}$ In Muslim societies, childlessness can be especially distressing for infertile females because their religion and culture allow men to have more than one wife at the same time and a female's inability to conceive gives the men a good excuse to remarry. Men are often pressurized into a second or multiple marriages and this adds to the psychological problem for the females. ${ }^{8}$

Psychological issues and emotional stress are important factors that will impact QoL in infertile couples. It is commonly seen that men and women tend to have different psychological responses at the start of infertility treatment. ${ }^{9}$ Women who are infertile portray a higher level of emotional disturbance, lower self-esteem and are more dissatisfied with their lives; whereas infertile men have lower psychological distress and better QoL than their female counterparts. ${ }^{10,11}$ Although men and women responded to the stress quite differently, the functioning and QoL of these couples are greatly impaired following the diagnosis of infertility. ${ }^{12}$

\section{Infertility related issue in Malaysia}

Between 1970 and 2010, Malaysia's total fertility rate (TFR) showed a decline from 5 to 2.1 births per woman. The trend in postponement of marriage, later age of first child, higher divorce rate, high educational status for women, a modern society and high standard of living were shown to be the contributing factors. ${ }^{13}$

Although infertility is one of the health problems that may cause a psychosocial impact on the married couple; only a few relevant local study have explored the issue. Hazlina et al and Musa et al for instance found that the wives of the infertile couple suffered severe psychological distress than their husbands. ${ }^{14,15}$ They felt embarrassed, sad and guilty. A high percentage of them developed symptoms of anxiety and depression with various degree of severity. The emotional coping style they used was associated with greater stress in both gender. ${ }^{15}$

The main objective of this study is to determine gender differences on the impact of psychological distress and its associated risk factors among infertile couples attending fertility clinics; and explored the correlation with QoL. The QoL were not assessed in both previous studies. ${ }^{14-15}$

\section{METHODS}

\section{Participants and sampling methods}

This is a cross-sectional study conducted in the Department of Obstetrics and Gynaecology, Hospital
Sultan Ismail (HSI). HIS is a public hospital in Johor Bahru, the southern part of Peninsular Malaysia. The sample collection was conducted for 6 months, from $1^{\text {st }}$ June to $30^{\text {th }}$ November 2018. Subjects were recruited from fertility clinics and eligible couples were approached during their clinic visit. Case notes were reviewed to determine whether they fulfilled the inclusion and exclusion criteria. Cases were selected by systematic random sampling: the first case selection was based on the first subject registered on the day, followed by three alternate listed names, until the targeted sample size was reached. The inclusion criteria included couples or either spouse diagnosed with primary or secondary infertility (male or female infertility) by a reproductive specialist, were literate in English or Malay (Bahasa Malaysia) and willing to give informed consent. Those couples or either spouse who refused to provide informed consent, were illiterate in English or Malay and were diagnosed with severe mental illness (SMI) or organic mental disorder were excluded from the study.

\section{Measurements}

The selected respondents were briefed on the study background and objective of the study and given a subject information sheet. Once consent was obtained, they were enrolled into the study. Instruments used were: a questionnaire on sociodemographic data; the Depression, Anxiety and Stress Scale, 21-item version (DASS-21); and the Fertility Quality of Life (FertiQoL) questionnaire; all instruments were the Malay or Bahasa Malaysia versions. ${ }^{16,17}$ Clinical profile questionnaires were completed by the investigator using information from the case notes.

The DASS-21 is used to measure the emotional states of depression, anxiety and stress using a set of three selfreport subscales. Each of the subscales contains seven items. Scores for the relevant items are summed to calculate the scores for depression, anxiety and stress. ${ }^{18}$ The Malay version of the DASS-21 is a valid instrument for use in a Malaysian population in view of its well documented reliability and its validation on various Malay samples, including clinical and non-clinical populations. $^{16}$

The FertiQoL questionnaire comprises 36 items that assess core (24 items) and treatment related QoL (10 items) and overall life and physical health (2 items). ${ }^{19}$ The average FertiQol across all domains can be determined by looking at the core FertiQoL score whereas the average QoL across treatment domains is determined by the treatment FertiQoL score. The core FertiQoL consists of four sub-scales (mind-body; emotional; relational; social) and the treatment FertiQoL has two subscales (treatment environment; treatment tolerability). The 36 FertiQoL items were rated by five response scales. High sub-scale and total scores suggest a better QoL. In order to calculate the raw scores, all items that belong to the sub-scales or total scale should be 
summed. Farnanaza et al found that the Malay version of the FertiQoL instrument has good validation indexes. ${ }^{17}$

\section{Sample size determination}

The sample size estimation was calculated using a twopopulation mean formula. ${ }^{20}$ Previous data indicate that the mean infertility specific QoL in infertile couples is 54.39 for females $(\mathrm{SD}=13.52)$ and 60.63 for males $(\mathrm{SD}=$ 14.07). ${ }^{21}$ Thus, a minimum sample size of 77 samples per group is needed to be able to reject the null hypothesis with probability (power) 0.8 . The type I error probability associated with this test of null hypothesis is 0.05 . With a $30 \%$ dropout rate, the sample size is 100 samples per group, with a total sample of 200 .

\section{Statistical analysis}

All data analyses were conducted using the International Business Machines (IBM®) Statistical Package for Social Sciences (SPSS $®$, version 24). Continuous variables were described as the mean and standard deviation whereas categorical variables were described as frequency and percentage. An independent t-test was used to evaluate the different scores of anxiety, depression and stress among wives and husbands and also to assess gender differences in the scores of QoL and psychological distress/ multivariate analyses were used to identify independent predictors.

\section{RESULTS}

\section{Socio-demographic profile of participants}

A total of 200 subjects (100 couples) were enrolled in this study, aged 23-44 years. The average ages (SD) of men and women were 33.6 (4.29) and 31.3(4.03) years, respectively. The demographic data also indicated that most of the respondents $(60.5 \%)$ were aged $31-40$ years, $34.5 \%$ were less than 31 years and only $5 \%$ were more than 40 years old. In terms of ethnic group distribution, majority of the subjects were Malay (93\%), followed by Indians (4\%), Chinese (2\%) and others (1\%).

More than half $(57.5 \%)$ of the subjects received tertiary education, followed by secondary education (38.5\%), primary education $(3.5 \%)$ and $0.5 \%$ without any formal education. The majority $(85.5 \%)$ of the respondents in this study were in full-time employment and the rest were housewives. Of the respondents, $66 \%$ had been married for 5 years or more and $34 \%$ had been married for less than 5 years.

Table 1: The FertiQoL scores by gender.

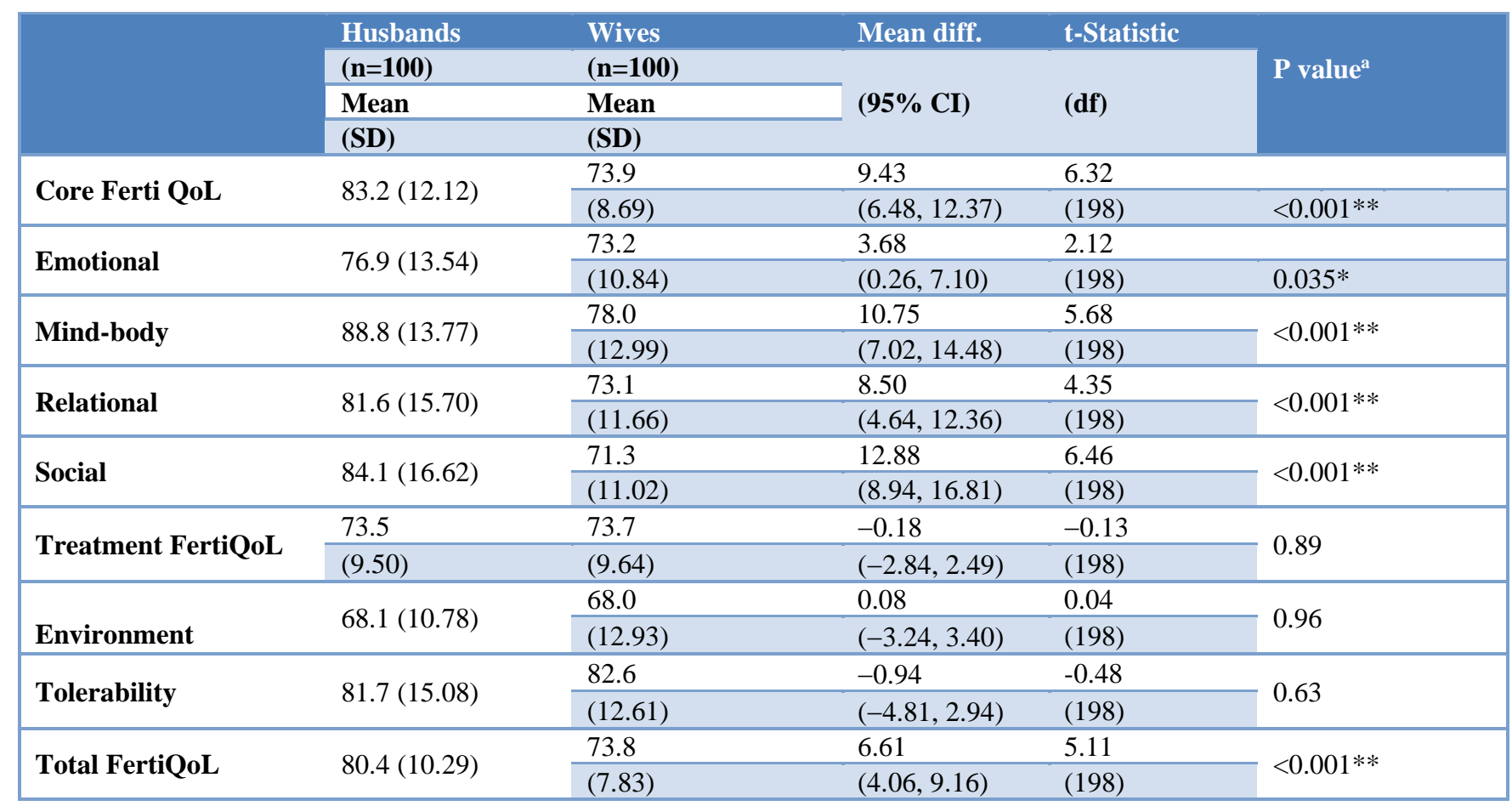

${ }^{a}$ Independent t-test: ${ }^{*} \mathrm{p}<0.05 ; * * \mathrm{p}<0.001$

\section{Clinical characteristics of participants}

The average (SD) duration of infertility was found to be $3.9(1.96)$ years. This study showed that females were the most common source of infertility $(38 \%)$, with $25 \%$ due to males, $16 \%$ due to both and $21 \%$ were non- established/unexplained cause of infertility. Majority of the participants had primary infertility (90\%) and the rest had secondary infertility. In addition, a large majority $(85 \%)$ of subjects had never tried fertility treatment or intrauterine insemination (IUI) at the time of inclusion in the study. 


\section{Gender difference in the DASS-21 scores}

A significant difference was detected between husbands and wives on the scores of all three sub-scale of DASS21. Wives reported higher levels of depression $(\mathrm{p}<0.001)$, anxiety $(\mathrm{p}<0.001)$ and stress $(\mathrm{p}<0.001)$ as compared to the husbands. Comparing the average (SD) scores for husbands and wives: depression scores were 5.9(4.07) and 8.8(4.57), anxiety scores were 6.6(4.36) and 8.6(4.03) and stress scores were 8.0(6.36) and 13.0(7.49), respectively.

\section{Gender difference in the FertiQoL scores}

Table 1 shows the FertiQoL results according to gender. The total FertiQoL scores were significantly higher for the husbands than the wives $(\mathrm{p}<0.001)$ : the mean \pm SD score of the total FertiQoL for the husbands was $80.4 \pm 10.29$, which is significantly higher than for the wives $(73.8 \pm 7.83 ; \mathrm{p}<0.001)$. The mean \pm SD score of the core FertiQoL for the husbands was $83.2 \pm 12.12$, which also was significantly higher than for the wives (73.9 $\pm 8.96 ; \mathrm{p}<0.001)$. The husbands showed significantly higher scores in the majority of the core FertiQoL domains, including emotional $(76.9 \pm 13.54$ vs. $73.2 \pm 10.84 ; \mathrm{p}=0.035)$, mind-body $(88.8 \pm 13.77$ vs. $78.0 \pm 12.99 ; \quad \mathrm{p}<0.001)$, relational $(81.6 \pm 15.70$ vs.
$73.1 \pm 11.66 ; \quad \mathrm{p}<0.001)$ and social $(84.1 \pm 16.62$ vs. $71.3 \pm 11.02 ; \mathrm{p}<0.001)$, compared to the wives. However, the score was not statistically significant in the other two domains.

\section{Multivariate analysis of factors associated with the DASS-21}

Simple linear regression (SLR) was performed against 8 variables prior to multiple linear regression (MLR) for all three DASS-21 sub-scales: anxiety, stress and depression. For the anxiety sub-scale, gender $(\mathrm{p}<0.001)$ and types of infertility $(\mathrm{p}=0.032)$ were significant. It shows a significant association between anxiety, gender and types of infertility (Table 2). For the stress sub-scale, gender $(p<0.001)$ and duration of infertility $(p=0.036)$ were significant. A significant association was found between stress, gender and duration of infertility (Table 3). For the depression sub-scale, gender $(\mathrm{p}<0.001)$, duration of marriage $(p=0.031)$ and duration of infertility $(p=0.043)$ were significant. There was a significant association between depression, gender, duration of marriage and duration of infertility among the infertile couples (Table 4). It seems that gender (female) was the only significant variable associated with all three DASS-21 subscale scores. Hence the wives are at higher risk of developing anxiety, depression and stress.

Table 2: Final regression analysis model of factors associated with the DASS-21 anxiety subscale as dependent variables.

\begin{tabular}{|c|c|c|c|c|c|}
\hline \multirow{2}{*}{ Variables } & \multicolumn{2}{|l|}{$\mathbf{S L R}^{\mathbf{a}}$} & \multicolumn{2}{|l|}{ MLR $^{\mathbf{b}}$} & \multirow[b]{2}{*}{$P$ value } \\
\hline & $b^{c}(95 \% C I)$ & $P$ value & Adj. $b^{d}(95 \% C I)$ & t-stat & \\
\hline Gender & $1.980(0.809,3.151)$ & $<0.001 * *$ & $1.231(0.819,3.141)$ & 3.362 & $<0.001 * *$ \\
\hline Types of Infertility & $2.011(0.026,3.996)$ & $0.032 *$ & $2.141(0.076,3.947)$ & 2.049 & $0.042 *$ \\
\hline
\end{tabular}

$\mathrm{R} 2=0.073$. Adjusted R2 $=0.064 ; \mathrm{F}=7.752$, SLR- Simple linear regression, MLR -Multiple linear regression, Significant $\mathrm{p}$-value, ${ }^{*} \mathrm{p}<0.05 ;{ }^{*} \mathrm{p}<0.001,{ }^{\mathrm{c} C r u d e}$ regression coefficients, ${ }^{\mathrm{d}}$ Adjusted regression coefficients, the model reasonably fits well. Model assumptions are met. There are no interaction between independent variables and no multi-collinearity problem

Table 3: Final regression analysis model of factors associated with the DASS-21 stress subscale as dependent variables.

\begin{tabular}{|c|c|c|c|c|c|}
\hline \multirow{2}{*}{ Variables } & \multicolumn{2}{|l|}{$\mathbf{S L R}^{\mathbf{a}}$} & $\mathbf{M L R}^{\mathbf{b}}$ & \multirow[b]{2}{*}{ t-stat } & \multirow[b]{2}{*}{$P$ value } \\
\hline & $b^{c}(95 \% C I)$ & $P$ value & Adj. $b^{d}(95 \% C I)$ & & \\
\hline Gender & $5.010(3.072,6.948)$ & $<0.001 * *$ & $2.326(1.769,4.171)$ & 4.875 & $<0.001 * *$ \\
\hline Duration of infertility & $2.338(0.286,7.101)$ & $0.036 *$ & $1.325(0.168,6.125)$ & 2.882 & $0.041 *$ \\
\hline
\end{tabular}

$\mathrm{R} 2=0.132$. Adjusted R2 $=0.123 ; \mathrm{F}=14.928$, SLR- Simple linear regression, MLR -Multiple linear regression, Significant $\mathrm{p}$-value, ${ }^{*} \mathrm{p}<0.05 ;{ }^{*} \mathrm{p}<0.001,{ }^{\mathrm{c} C r u d e}$ regression coefficients, ${ }^{\mathrm{d}}$ Adjusted regression coefficients, the model reasonably fits well. Model assumptions are met. There are no interaction between independent variables and no multi-collinearity problem

\section{Multivariate analysis of factors associated with total FertiQoL}

SLR was performed prior to MLR analysis revealed that six of eight variables were found to be significant: gender $(p<0.001)$, duration of marriage $(p=0.045)$, duration of infertility ( $p=0.036)$, causes of infertility ( $p=0.016)$, types of infertility $(\mathrm{p}=0.020)$ and previous history of IUI
( $p=0.028)$. The causes of infertility were classified into two groups: male and non-male factors. Non-male factor include female causes and non-established/ unexplained causes of infertility. Table 5 shows MLR analysis of the total FertiQoL scores and its associated factors. Gender $(\mathrm{p}<0.001)$ causes of infertility $(\mathrm{p}=0.004)$, types of infertility $(\mathrm{p}=0.022)$ and previous history of receiving IUI ( $p=0.020$ ) were found to be significantly associated with QoL of the infertile couples. 
Table 4: Final regression analysis model of factors associated with the DASS-21 depression subscale as dependent variables.

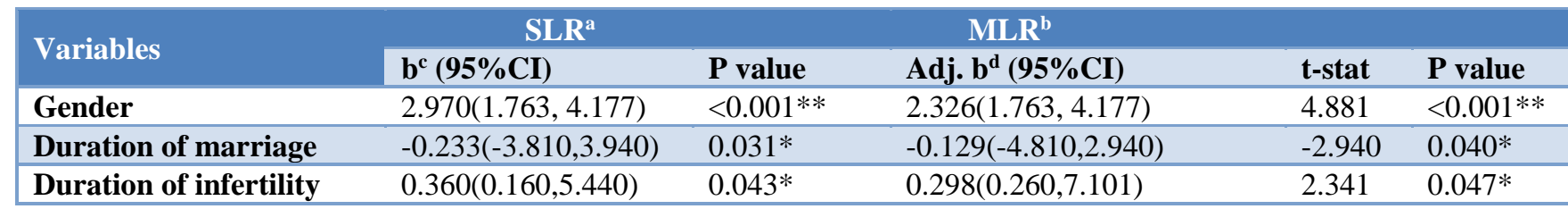

$\mathrm{R} 2=0.120$. Adjusted R2 $=0.106 ; \mathrm{F}=8.906$, SLR- Simple linear regression, MLR -Multiple linear regression, Significant $\mathrm{p}$-value, ${ }^{*} \mathrm{p}<0.05 ; * \mathrm{*} p<0.001$, ${ }^{\mathrm{c} C r u d e}$ regression coefficients, ${ }^{\mathrm{d}}$ Adjusted regression coefficients, The model reasonably fits well. Model assumptions are met. There are no interaction between independent variables and no multi-collinearity problem

Table 5: Final regression analysis model of factors associated with the total FertiQoL as dependent variables.

\begin{tabular}{|c|c|c|c|c|c|}
\hline \multirow{2}{*}{ Variables } & \multicolumn{2}{|l|}{$\mathbf{S L R}^{\mathbf{a}}$} & $\mathbf{M L R}^{\mathbf{b}}$ & \multirow[b]{2}{*}{$t$-Stat. } & \multirow[b]{2}{*}{$P$ value } \\
\hline & $b^{c}(95 \%$ CI $)$ & $P$ value & $\operatorname{Adj} . b^{\text {d }}(95 \%$ CI $)$ & & \\
\hline Gender & $-6.606(-9.132,-4.080)$ & $<0.001 * *$ & $-6.606(-9.155,-4.057)$ & -5.158 & $<0.001 * *$ \\
\hline Duration of Marriage & $-0.136(-4.660,3.940)$ & $0.045^{*}$ & $-3.071(-6.566,0.796)$ & -1.570 & 0.118 \\
\hline Duration of Infertility & $2.236(-1.545,5.142)$ & $0.036 *$ & $0.116(-1.545,2.017)$ & 1.167 & 0.245 \\
\hline Causes of Infertility & $0.198(-1.200,8.003)$ & $0.016^{*}$ & $0.197(-1.200,8.223)$ & 3.909 & $0.004 *$ \\
\hline Types of Infertility & $-4.240(-8.834,9.353)$ & $0.020 *$ & $-3.550(-8.866,9.656)$ & -3.312 & $0.022 *$ \\
\hline Previous history of IUI ${ }^{e}$ & $2.168(0.216,5.317)$ & $0.028 *$ & $2.199(0.216,5.317)$ & 3.339 & $0.020 *$ \\
\hline
\end{tabular}

$\mathrm{R} 2=0.081 ;$ adjusted $\mathrm{R} 2=0.057 ; \mathrm{F}=3.408 ; * \mathrm{p}<0.05 ; * * \mathrm{p}<0.001$, ${ }^{\mathrm{S}} \mathrm{SLR}$, simple linear regression; ${ }^{\mathrm{B}} \mathrm{MLR}$, multiple linear regression; ${ }^{c}$ crude regression coefficient; dadjusted regression coefficient; eintrauterine insemination, The model fits reasonably well and the model assumptions are met. There are no interactions between independent variables and no multicollinearity problems.

\section{DISCUSSION}

\section{Socio-demographic distribution}

A total of 200 respondents (100 couples) participated in this study, with a response rate of $100 \%$. The proportion of each race was consistent with the Malaysian population, especially the ethnic distribution in Johor Bahru district, which showed that Malays recorded the highest numbers of the total population. ${ }^{22}$ The demographic data also indicated that most of the respondents $(60.5 \%)$ were aged between 31 and 40 years, with $34.5 \%$ of respondents aged less than 31 years and only $5 \%$ aged more than 40 years. With regard to the clinical characteristics, $66 \%$ of respondents had been married for 5 years: half of them had infertility problems for 5 years or more but a large majority did not receive any previous fertility treatment. These figures were consistent with a previous local study. ${ }^{14}$

\section{Risk factors and vulnerability to psychological distress}

The main findings of the study were that among infertile Malaysian couples, wives significantly $(p<0.001)$ reported higher levels of depression, anxiety and stress. The findings are consistent with previous local studies and other Western studies showing that wives of infertile couples are more vulnerable to psychological distress such as depression, anxiety and emotional unstable than their husbands. ${ }^{14,15,23,24}$ We found that female gender (wives) was the only variable significantly associated with the severity of all three DASS-21 sub-scale scores of depression, anxiety and stress $(\mathrm{p}<0.001)$; and considered as risk factor for psychological distress development. Types and causes of infertility partially contributed to the scores.

\section{Gender differences on the impact of psychological distress and QoL}

We found that generally women had lower QoL than men. The total FertiQoL scores were significantly higher in the husbands than the wives $(\mathrm{p}<0.001)$. This finding was similar to the results of another Asian country: a study conducted among Taiwanese infertile couples showed that men had significantly higher core FertiQoL sub-scale scores of the emotional and mind-body domains. ${ }^{21}$ The male spouses in our study were shown to have a higher score in the emotional and mind-body domains than the females, indicating that infertile women in Malaysia may experience greater emotional stress and have poorer physical health status. This finding is compatible with a previous study conducted in Iran, where a higher depression rate was found in infertile women compared to their husbands. ${ }^{25}$

Another study from Japan reported that severe depression and anxiety in infertile women may result from a lack of support from their spouses and feelings of stress. ${ }^{26}$ This study found that age was not related to the severity of depression, anxiety and stress of the participants, consistent with a study from Pakistan. ${ }^{27}$ 


\section{Quality of life and its associated factors}

The MLR results showed a significant association between gender, causes of infertility, types of infertility and previous history of IUI with QoL of the infertile couples. Further analysis showed that the total FertiQoL had a lower score when the male partners contributed to the causes of infertility. The impact of male infertility on the QoL of couples has been evaluated by other investigators. ${ }^{28}$ They found that male infertility had lesser negative effects on QoL and that the FertiQoL scores were lower in males than their infertile spouses. Other studies also suggested that infertile women had a lower QoL and were at increased risk of developing emotional problems compared to their partners, regardless of the causes of infertility. ${ }^{29}$

There were several limitations of this study. First, the weakness of the cross-section study design. It cannot determine the exact cause or psychological distress and factors correlated to psychological distress and QoL among the infertile couples. The best study design is a longitudinal case-control study. Secondly, a small sample size which involved one study centre only. It did not represent the whole population of infertile couples in Malaysia. There is possibility that a different result could be obtained if the study were repeated in private hospital or other location of the country. Thirdly, all the two questionnaires used in this study were self-reported questionnaires. The response may be influenced by the participants' own subjective perception. It is better to have questionnaires with more objective assessment (e.g. clinician rated). Lastly, there are several confounding variables which are not controlled due to time limitation and lack of human resources. This included coping skills, pre-existing marital relationship and the subject's personality trait. For examples good coping skills may reduce the severity of depression, anxiety and stress.

\section{CONCLUSION}

The current findings suggest that clinicians working with infertility couples should be aware that the wives were more susceptible to develop psychological distress and had lower QoL. Female gender (wives), couples with a longer duration of infertility, primary infertility, male cause of infertility and history of IUI are at higher risks for development of severe psychological distress and low QoL. Emotional distress also caused the couples to withdraw from infertility treatment, lead to high failure rate and further threatening their QoL.

\section{ACKNOWLEDGMENTS}

Authors would like to thank all staff of Department of Psychiatry, Hospital Sultan Ismail, Johor Bahru who were involved in the study.
Funding: No funding sources

Conflict of interest: None declared

Ethical approval: The study was approved by the Institutional Ethics Committee of Universiti Teknologi MARA (UiTM), Medical Research and Ethics Committee (MREC) of Ministry of Health Malaysia (Protocol No: NMRR-18-554-40676) and the Clinical Research Centre (CRC) of Hospital Sultan Ismail, Johor Bahru, Malaysia

\section{REFERENCES}

1. Zegers-Hochschild F, Adamson, GD, de Mouzon J, Ishihara O, Mansour R, Nygren K, et al. The International Committee for Monitoring Assisted Reproductive Technology (ICMART) and the World Health Organization (WHO) Revised Glossary of ART Terminology, 2009. Hum Reprod. 2009;24(11):2683-7.

2. Skevington SM, Lotfy M, O'Connell KA. World Health Organisation's WHOQOL-BRIEF quality of life assessment: psychometric properties and results of the international field trial. A report from the WHOQOL. Qual Life Res. 2004;13:299-310.

3. Maruani P, Schwartz D. Sterility and fecundability estimation. J Theor Biol. 1983;105(9):211-9.

4. Sexton MB, Byrd MR, Von Kluge S. Measuring resilience in women experiencing infertility using the CD-RISC: Examining infertility-related stress, general distress, and coping styles. J Psychiatr Res. 2010;44(4):236-41.

5. Alhassan A, Ziblim A, Muntaka S. A survey on depression among infertile women in Ghana. BMC Womens Health. 2014;42:14.

6. Kucur Suna K, Ilay G, Aysenur A, Kerem Han G, Eda Ulku U, Pasa U, et al. Effects of infertility etiology and depression on female sexual function. J Sex Marital Ther. 2016;42(1):27-35.

7. Aarts JWM, Van Empel IWH, Boivin J, Nelen WL, Kremer JAM, Verhaak CM. Relationship between quality of life and distress in infertility: A validation study of the Dutch FertiQoL. Hum Reprod. 2011;26(5):1112-8.

8. Dyer S. The value of children in African countries: insights from studies on infertility. J Psychosom Obstet Gynaecol. 2007;28(2):69-77.

9. Connolly KJ, Edelmann RJ, Cooke ID, Robson J. The impact of infertility on psychological functioning. J Psychosom Res. 1992;36(5):459-68.

10. Greil AL. Infertility and psychological stress, A critical review of the literature. Soc Sci Med. 1997;45(11):1679-704.

11. Newton CR. The fertility problem inventory: Measuring perceived infertilty-related stress problem. Fertil Steril. 1999;77(6):1233-40.

12. Luk BHK, Loke AC.The impact of infertility on the psychological well-being, marital relationships, sexual relationships, and quality of life of couples: A systematic review. J Sex Marital Ther. 2015;41(6):610-25. 
13. Rashid SA, Ghani AP, Daud N, Azemi SNAN, Hilmi ZAG, Razak MR, et al. Fertility dynamics in Malaysia: comparison of Malays, Chinese and Indian ethnics. In 3rd International Conference on Education and Social Sciences (INTCESS 2016). 8th to 10th February 2016, Istanbul, Turkey. Available at: http://www.ocerint.org/intcess16/.

14. Hazliza NNH, Norliza M, Bahari SI, Hasanah CI. Etiological factors and psychosocial impacts of female partner in couples with infertility. Int Med J. 2006;13(1):59-65.

15. Musa R, Ramli R, Yazmie AWA, Khadijah MBS, Hayati MY, Midin M, et al. A preliminary study of the psychological differences in infertile couples and their relation to the coping styles. Compr Psychiatry. 2014;55(Suppl. 1):S65-9.

16. Musa R, Ariff MF, Zaini Z. Translation, validation and psychometric properties of Bahasa Malaysia version of the Depression Anxiety and Stress Scales (DASS). Asean J Psychiatr. 2007;8(2):82-9.

17. Farnanza A, Suzanna D, Zaliha I,Rozman R, Ani ZA. Validation of Malay version Fertility Quality of Life (FertiQoL). J Clin Health Sci. 2017;2(Suppl 2).

18. Lovibond PF. Long-term stability of depression, anxiety, and stress syndromes. J Abnorm Psychol. 1998;107(3):520-2.

19. Boivin J, Takefman J, Braverman A. The fertility quality of life (FertiQoL) tool: Development and general psychometric properties. Hum Reprod. 2011;26(8):2084-91.

20. Lemeshow S, Hosmer DW, Klar J, Lwanga SK, World Health Organization. Adequacy of sample size in health studies. Chichester: Wiley; 1990.

21. Hsu PY, Lin MW, Hwang JL, Lee MS, Wu MH. The fertility quality of life (FertiQoL) questionnaire in Taiwanese infertile couples. Taiwan J Obstet Gynecol. 2013;52:204-9.

22. Population Quick Information, Department of Statistic Malaysia, Kuala Lumpur; 2018. Available at: https://www.pqi.stats.gov.my. Assessed 2020 April 26.

23. Guerra D, Llobera A, Veiga A, Barri PN. Psychiatric morbidity in couples attending a fertility service. Human Reproduction. Hum Reprod. 1998;13(6):1733-6.

24. Tarlatzis I, Tarlatzis BC, Diakogiannis I, Bontis J, Lagos S, Gavriilidou D, Mantalenakis S. Psychosocial impacts of infertility on Greek couples. Hum Reprod. 1993;8(3):396-401.

25. Ashkani H, Akbari A, Heydari S. Epidemiology of depression among infertile and fertile couples in Shiraz, Southern Iran. Indian J Med Sci. 2006;60(10):399-406.

26. Matsubayashi H, Hosaka T, Izumi SI, Suzuki T, Kondo A, Makino T. Increased depression and anxiety in infertile Japanese women resulting from lack of husband's support and feelings of stress. Gen Hosp Psychiatry. 2004;26(5):398-404.

27. Yusuf L. Depression, anxiety and stress among female patients of infertility; A case control study. Pak J Med Sci. 2016;32(6):1340-3.

28. Malik S, Coulson N. The male experience of infertility: a thematic analysis of an online infertility support group bulletin board. J Reprod Infant Psychol. 2008;6(1):18-30.

29. Huppelschoten AG, Van Dongen AJ, Verhaak CM, Smeenk JM, Kremer JA, Nelen WL. Differences in quality of life and emotional status between infertile women and their partners. Human Reprod. 2013;28(8):2168-76.

Cite this article as: Ibrahim N, Mohamed S, Ismail Z, Salleh MR. Psychological distress and quality of life of Malaysian infertile couples. Int J Reprod Contracept Obstet Gynecol 2021;10:61-7. 